Supporting information for

\title{
Revealing Electrical Double Layer Potential of Substrates by Hysteresis Ion Transport in Scanning Ion Conductance Microscopy
}

Yingfei Ma, Dengchao Wang*

School of Chemical Sciences, University of Chinese Academy of Sciences, Beijing, 10049, P. R. China

*Email: wangdengchao@ucas.ac.cn

\section{Table of contents}

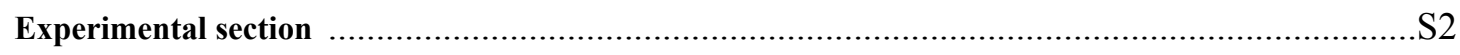

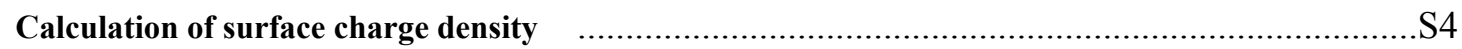

Figure S1 (A) Representative TEM image and (B) I-V curve of the prepared quartz nanopipette.............S5

Figure S2 Experimental I-V curves of nanopipettes in bulk solution .........................................S6

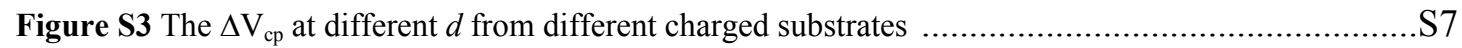

Figure S4 Normalized enclosed charges in the I-V loops at high and low conductivity states................S8

Figure S5 (A) Approach curve of SICM and (B) Current image of substrate .................................S9

Figure S6 The nanoscale surface potential imaging of positive charged glass substrate ....................S10 


\section{Experimental section}

Chemicals and Materials. All solutions were prepared with ultrapure water (Milli-Q, $18.2 \mathrm{M} \Omega \bullet \mathrm{cm}$, total organic content $<10 \mathrm{ppb})$. Potassium chloride $(\mathrm{KCl}, \geq 99.5 \%)$ was purchased from Macklin and used as received without further purification. Positive and regular glass slides were used as substrates, and they were washed thoroughly with ethanol and ultrapure water before use.

Fabrication of nanopipettes. The nanopipettes with $50 \sim 80 \mathrm{~nm}$ radius $(a)$ were produced by pulling quartz capillaries (o.d. $1.0 \mathrm{~mm}$, i.d. $0.5 \mathrm{~mm}$, Sutter Instrument Company) with a laser pipette puller (P-2000, Sutter Instruments; pulling parameters: Line 1: Heat 650, Fil 3, Vel40, Del 180, Pul 100). The fabricated nanopipettes were characterized by transmission electron microscopy (TEM, JEOL TEM-2100 Instrument) and ion conductivity measurements. In conductivity experiments, an electrolyte-filled nanopipette was immersed in a same bath electrolyte solution, the potentials were applied between two home-made $\mathrm{Ag} / \mathrm{AgCl}$ electrodes placed back-inserted the pipette and in the bulk solution.

Scanning Ionic Conductance Microscopy (SICM). All SICM measurements were performed in a Faraday cage with a home-built SICM device mounted on an optical vibration isolation table (Zolix, ZTP10-80), as described in our previous report ${ }^{1}$. Briefly, the nanopipette was mounted perpendicularly on a piezoelectric positioning stage (XYZ, P-621.2CD, P-621.ZCD, Physik Instrument), controlled by an E-727 digital piezo controller (Physik Instrument), which, in turn, was controlled from a PC running Labview 2019 (National Instruments). Under closed-loop mode, the X, Y, Z piezoelectric positioners would provide a high resolution of $0.4 \mathrm{~nm}$ and a total travel range of $100 \mu \mathrm{m}$, respectively. The piezoelectric positioning stage was attached to a 3-axis manual linear stage (Zolix, SR13-4016SL) for coarse positioning. A Z-axis manual linear stage with the object of interest was also mounted on the optical table. A long-distance video microscope (SN-108030C) was used to monitor the tip-substrate distance. A CHI 760E bipotentiostat ( $\mathrm{CH}$ Instruments) was employed for electrochemical operations.

For approach curve measurements, a nanopipette probes was initially positioned a few tens of micrometers above the substrate surface by adjusting the manual linear stage, monitored with the video microscope. After applying voltages between the $\mathrm{Ag} / \mathrm{AgCl}$ electrodes inside and outside the nanopipette (positive voltage is defined as inside vs. outside), the Z-axis piezoelectric actuator was used to move the tip toward the substrate at a speed of $\sim 50 \mathrm{~nm} / \mathrm{s}$. The speed would be slow down to $2 \mathrm{~nm} / \mathrm{s}$ when the tip was getting close to the substrate, as indicated by the drastically changed current (i.e. $10 \%$ change in amplitude). The approach curves were then fitted by numerical simulation to obtain the accurate separation distance $(d)$ values.

At selective separation distances, the current-potential $(I-V)$ measurements were performed at a scan rate of $0.1 \mathrm{~V} / \mathrm{s}$, the sampling interval was $1 \mathrm{mV}$ with a filter frequency of $150 \mathrm{~Hz}$. The I-V curves were repeated at least 8 times to get the averaged cross point potential $\left(\mathrm{V}_{\mathrm{cp}}\right)$. For the surface potential imaging by $\mathrm{V}_{\mathrm{cp}}$, the nanopipettes was placed above the substrate $(d=\sim 10 \mathrm{~nm})$. Once the $I-V$ curve was recorded, the $\mathrm{X}$, Y-axis piezoelectric actuators controlled the tip moved to next position for next measurements, each step for $\mathrm{X}$ and $\mathrm{Y}$ direction was $100 \mathrm{~nm}$.

Numerical Simulation. The pinched hysteresis ionic current responses of nanopipette near charged substrate were also simulated by the finite element analysis using commercial software COMSOL Multiphysics 5.4. Basically, 2-D axisymmetric model with "transport of dilute species" and "electrostatic modules" were used, following previous reports ${ }^{2}$. The mass transport processes in nanopipettes are described by the Nernst-Planck equation,

$$
J_{i}=-D_{i} \nabla_{i}-z_{i} \frac{D_{i}}{R T} F c_{i} \nabla \emptyset
$$


Where $J_{i}$ is the total flux of species $i, D_{i}$ is the diffusion coefficient of species $i, R$ is the universal gas constant, $T$ is the temperature (which was set to $293.15 \mathrm{~K}$ ). The Poisson equation was used to calculate the potential distribution,

$$
\nabla^{2} \emptyset=\frac{-F}{\varepsilon \varepsilon_{0}} \sum_{i} Z_{i} c_{i}
$$

Where $F$ is the Faraday constant, $\varepsilon$ is the relative permittivity of water (which was set to 80 ), $\varepsilon_{0}$ is the vacuum permittivity, and $z_{i}$ is the charge on species $i\left(+1\right.$ for $\mathrm{K}^{+}$and -1 for $\left.\mathrm{Cl}^{-}\right)$. A time-dependent triangular potential waveform is applied (scan rate $=v$ ), and the resulting current responses can be obtained by surface integration of the total ion fluxes at the electrode surface. 


\section{Calculation of surface charge density}

With Gouy-Chapman (GC) model $^{3}$, the surface charge density $\sigma^{M}$ can be evaluated by following equation for dilute aqueous solutions at $25^{\circ} \mathrm{C}$

$\sigma^{M}=11.7 C^{* 1 / 2} \sinh \left(19.5 z \phi_{0}\right)$

Where, $C^{*}$ is the bulk z:z electrolyte concentration in mol/L (which was $0.01 \mathrm{~mol} / \mathrm{L}$ ), $z=1$ is the ion charge, $\phi_{0}=-40 \mathrm{mV}$ is the potential at $\mathrm{x}=0$ relative to the bulk solution, and the calculated $\sigma^{M}$ is $-0.01 \mathrm{C} / \mathrm{m}^{2}$, which agrees very well to the typical value for glass substrates.

$\sigma^{M}=11.7 *(0.01)^{1 / 2} \sinh (-19.5 * 1 * 40 \mathrm{mV})=-1 \mu \mathrm{C} / \mathrm{cm}^{2}=-0.01 \mathrm{C} / \mathrm{m}^{2}$

\section{References}

(1) Ma, Y.; Liu, R.; Shen, X.; Wang, D. Quantification of Asymmetric Ion Transport in Glass Nanopipettes near Charged Substrates. ChemElectroChem 2021, DOI: 10.1002/celc.202101180.

(2) Wang, D.; Brown, W.; Li, Y.; Kvetny, M.; Liu, J.; Wang, G. Correlation of Ion Transport Hysteresis with the Nanogeometry and Surface Factors in Single Conical Nanopores. Anal. Chem. 2017, 89, 11811-11817.

(3) Bard, A. J.; Faulkner, L. R. Double-Layer Structure and Adsorption. In Electrochemical Methods: Fundamentals and Applications, 2nd ed.; John Wiley \& Sons: 2001, pp 534-579. 

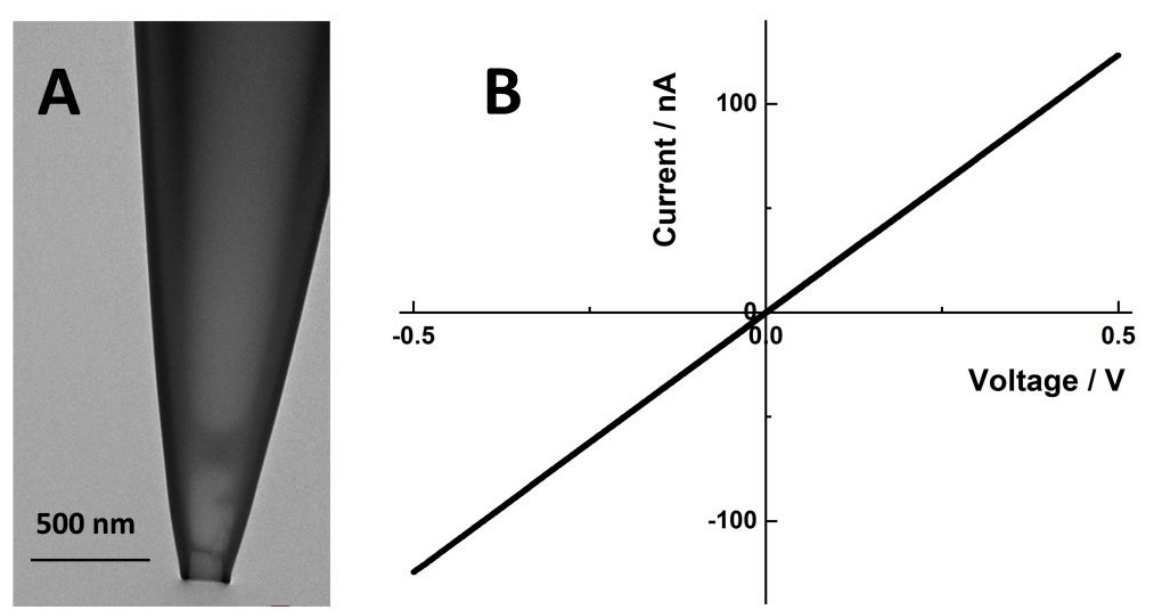

Figure S1. (A) TEM image and (B) I-V curve of the prepared quartz nanopipette.

The radius $(a)$, half-cone angle $(\theta)$ and RG value can be estimated from the TEM image. Electrochemical measurements were also used to estimate the nanopipette radius $(a)$ in concentrated electrolyte solutions. The radius can be calculated by using following equation (S4):

$a=\frac{(\pi \tan \theta)^{-1}+0.25}{R K}$

Where $\theta$ is the half-cone angle (the $\tan \theta$ was set to 0.12$), K$ is the solution conductivity $(0.1118 \mathrm{~S} / \mathrm{cm}$ in $1 \mathrm{M} \mathrm{KCl}$ solution), $R$ is the average resistance and could be calculated according to

$R=\frac{2 \boldsymbol{V}}{\left|\boldsymbol{i}_{+}\right|+\left|\boldsymbol{i}_{-}\right|}$

where $V$ is the voltage (which was set to $\pm 0.1 \mathrm{~V}$ ), $i_{+}$and $i_{-}$are the current at $+0.1 \mathrm{~V}$ and $-0.1 \mathrm{~V}$ (which are $24.49 \mathrm{nA}$ and $-25.61 \mathrm{nA}$, respectively), the estimate nanopipette radius is $65 \mathrm{~nm}$.

$a=\frac{(\pi \tan \theta)^{-1}+0.25}{R K}=\frac{\frac{1}{\pi \tan \theta}+0.25}{\frac{2 * 0.1 V * 0.1118 \mathrm{~S} / \mathrm{cm}}{|24.49 \mathrm{nA}|+|-25.61 \mathrm{nA}|}}=65 \mathrm{~nm}$ 

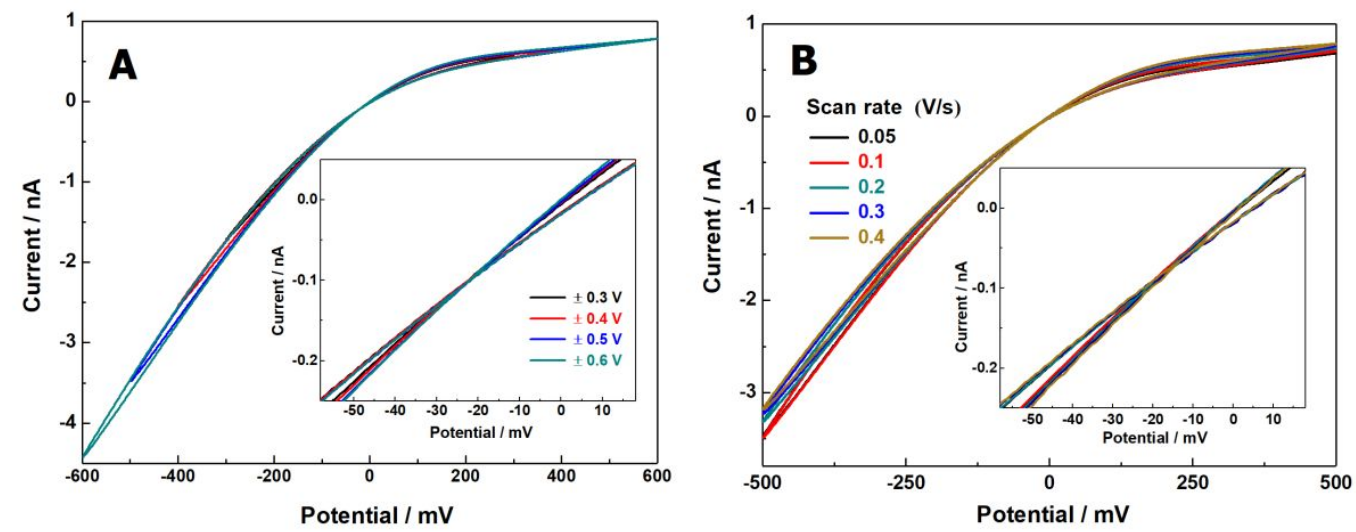

Figure S2. The experimental I-V curves from nanopipettes under different potential waveforms in $10 \mathrm{mM}$ $\mathrm{KCl}$ solution: (A) potential amplitudes and (B) scan rates. The constant cross point region is zoomed in as inset. $a=\sim 70 \mathrm{~nm}$. 

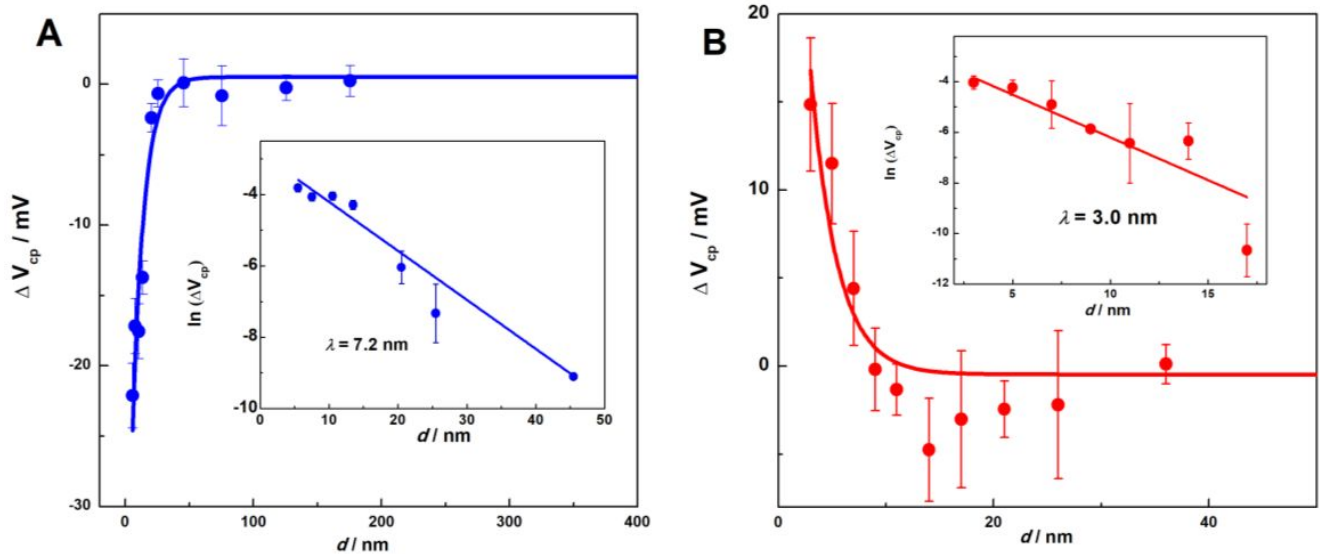

Figure S3. The $\Delta \mathrm{V}_{\mathrm{cp}}\left(\mathrm{V}_{\mathrm{cp}}-\mathrm{V}_{\mathrm{cp}, \infty}\right)$ at various $d$ from (A) negatively and (B) positively charged glass slides in $10 \mathrm{mM} \mathrm{KCl}$, and their fitting by exponential decay equation, with natural logarithm form shown as inset. 


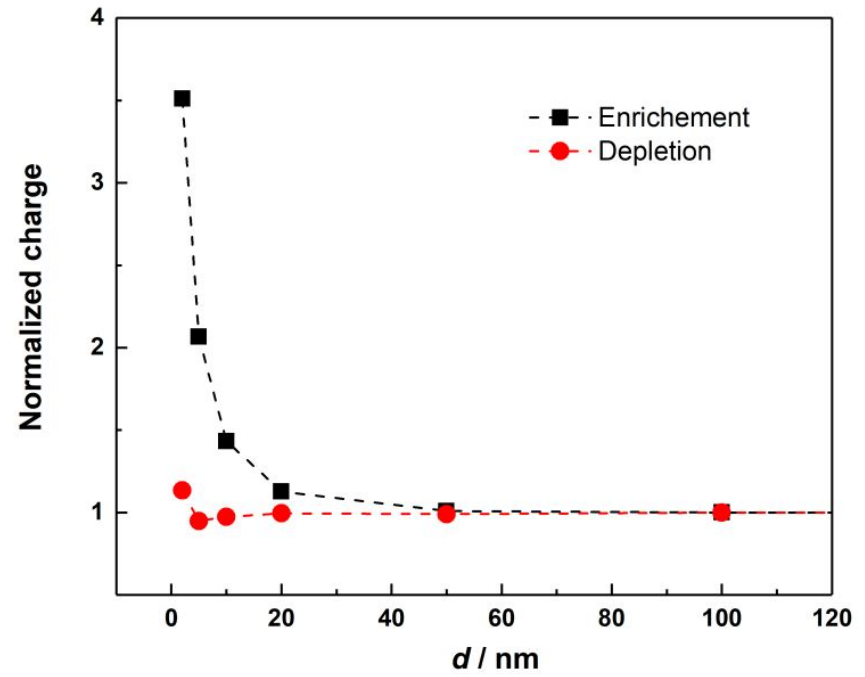

Figure S4 Normalized enclosed charges in the I-V loops at high (black) and low (red) conductivity states in Figure 3. 

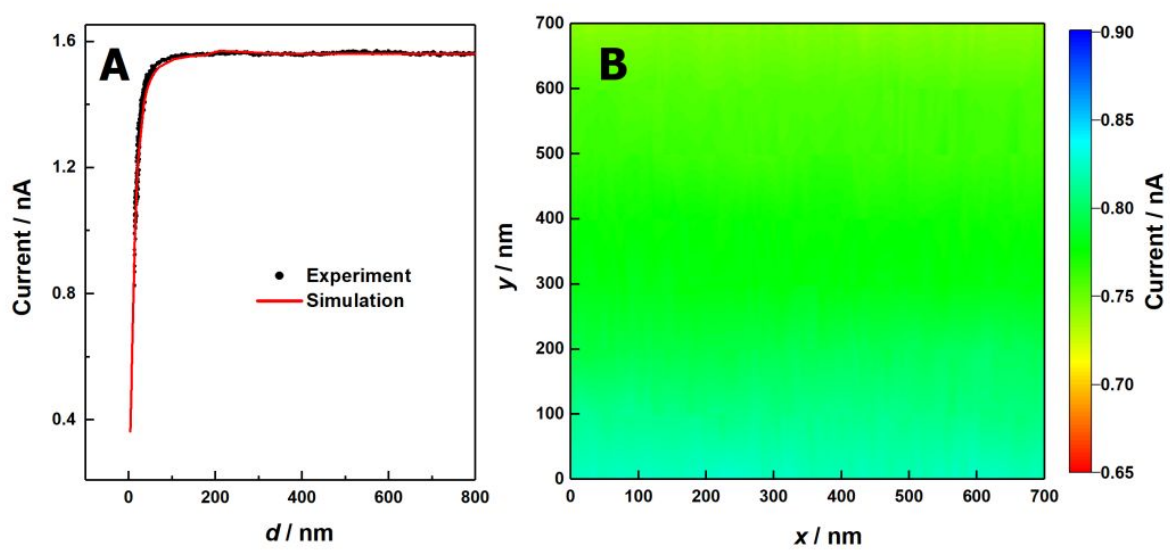

Figure S5. (A) The experimental (black dots) and simulated (red curve) approach curves of glass nanopipettes toward glass substrate at $+0.5 \mathrm{~V}$ in $10 \mathrm{mM} \mathrm{KCl}$ solution. Fitting conditions: $a=70 \mathrm{~nm}, \tan \theta=0.12, \mathrm{RG}=$ 1.2. $\sigma_{\text {pipette }}=\sigma_{\text {substrate }}=-0.01 \mathrm{C} / \mathrm{m}^{2}$, and (B) current amplitude imaging at $+0.5 \mathrm{~V}, d=\sim 11 \mathrm{~nm}$. 


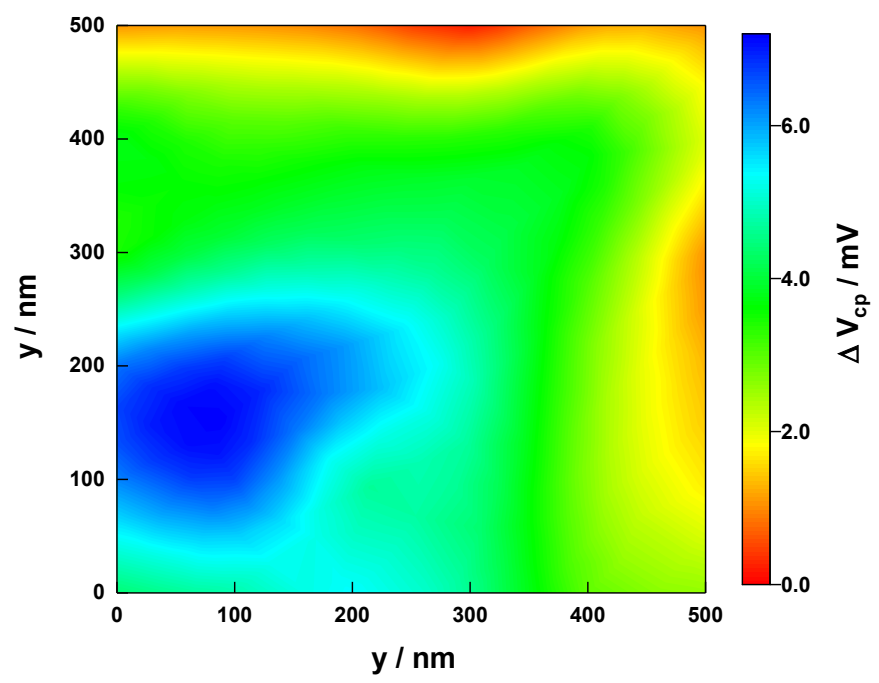

Figure S6. The nanoscale surface potential imaging of positively charged glass substrate based on $\mathrm{V}_{\mathrm{cp}}$, which were obtained by scanning I-V curves at $0.2 \mathrm{~V} / \mathrm{s}$ at respective positions. $a=52 \mathrm{~nm}, d=16 \mathrm{~nm}$, and $\mathrm{d} x=\mathrm{d} y=100$ $\mathrm{nm}$. 